\title{
Philosophiques
}

\section{Herméneutique cosmique}

\section{Brian McLaughlin}

Volume 27, numéro 1, printemps 2000

Le matérialisme contemporain

URI : https://id.erudit.org/iderudit/004956ar

DOI : https://doi.org/10.7202/004956ar

Aller au sommaire du numéro

\section{Éditeur(s)}

Société de philosophie du Québec

\section{ISSN}

0316-2923 (imprimé)

1492-1391 (numérique)

Découvrir la revue

\section{Citer cet article}

McLaughlin, B. (2000). Herméneutique cosmique. Philosophiques, 27(1), 63-76. https://doi.org/10.7202/004956ar

\section{Résumé de l'article}

L'herméneutique cosmique est le projet qui consiste à établir tous les faits de notre monde à partir d'un sous-ensemble propre de ces faits - soit des faits de base. Ce texte porte sur un projet spécifique d'herméneutique cosmique qui présuppose que tous les faits de notre monde sont déterminés par ses faits physiques. Suivant Frank Jackson, je formule cette thèse du déterminisme physique de la façon suivante : toute réplique physique minimale de notre monde en est une réplique simpliciter. Une réplique physique minimale de notre monde est un monde qui est exactement comme le nôtre sous tous ses aspects physiques, et qui ne contient rien de plus que ce qui est requis pour cela. Une réplique simpliciter de notre monde est un monde qui est exactement comme le nôtre sous tous ses aspects. Je me concentre sur la question épistémologique suivante : si la thèse du déterminisme physique est vraie, alors qu'est-ce qu'un démon laplacéen devrait connaître, en plus des faits physiques de notre monde, pour pouvoir prédire tous les faits de notre monde à partir des faits physiques?
Ce document est protégé par la loi sur le droit d'auteur. L’utilisation des services d’Érudit (y compris la reproduction) est assujettie à sa politique d'utilisation que vous pouvez consulter en ligne.

https://apropos.erudit.org/fr/usagers/politique-dutilisation/ 


\title{
Herméneutique cosmique
}

\author{
BRIAN MCLAUGHLIN \\ brianmc@rci.rutgers.edu \\ Department of Philosophy \\ Rutgers University
}

\begin{abstract}
RÉSUMÉ. - L'herméneutique cosmique est le projet qui consiste à établir tous les faits de notre monde à partir d'un sous-ensemble propre de ces faits - soit des faits de base. Ce texte porte sur un projet spécifique d'herméneutique cosmique qui présuppose que tous les faits de notre monde sont déterminés par ses faits physiques. Suivant Frank Jackson, je formule cette thèse du déterminisme physique de la façon suivante : toute réplique physique minimale de notre monde en est une réplique simpliciter. Une réplique physique minimale de notre monde est un monde qui est exactement comme le nôtre sous tous ses aspects physiques, et qui ne contient rien de plus que ce qui est requis pour cela. Une réplique simpliciter de notre monde est un monde qui est exactement comme le nôtre sous tous ses aspects. Je me concentre sur la question épistémologique suivante : si la thèse du déterminisme physique est vraie, alors qu'est-ce qu'un démon laplacéen devrait connaître, en plus des faits physiques de notre monde, pour pouvoir prédire tous les faits de notre monde à partir des faits physiques?
\end{abstract}

ABSTRACT. - Cosmic hermeneutics is the project of ascertaining all of the facts of our world from some proper subclass of the facts of our world - some class of ur-facts. I am concerned with a particular project of cosmic hermeneutics that presupposes that all the facts of our world are determined by the physical facts of our world. Following Frank Jackson, I formulate this thesis of physical determinism as follows: any minimal physical duplicate of the actual world is a duplicate simpliciter of the actual world. A minimal physical duplicate of our world is a world that is exactly like our world in every physical respect and that contains nothing more than is required to be exactly like our world in every physical respect. A duplicate simpliciter of our world is a world that is exactly like our world in every respect whatsoever. I focus on the following epistemological question: if physical determinism is true, then what, in addition to the physical facts of our world, would a Laplacean demon have to know in order to predict all of the facts of our world from the physical facts?

Pierre Laplace a caractérisé, de façon notoire, le déterminisme causal du mouvement relativement à ses conséquences épistémiques pour un observateur suffisamment bien informé qui disposerait d'une capacité de calcul infinie :

Une intelligence qui, pour un instant donné, connaîtrait toutes les forces dont la nature est animée et la situation respective des êtres qui la composent, si d'ailleurs elle était assez vaste pour soumettre ces données à l'Analyse, embrasserait dans la même formule les mouvements des plus grands corps de l'univers et ceux du plus léger atome: rien ne serait incertain pour elle, et I'avenir, comme le passé, serait présent à ses yeux ${ }^{1}$.

1. Laplace, 1886, p. vi-vii.

PHILOSOPHIQUES 27/1 — Printemps 2000, p. 63-76 
M ême si la thèse du déterminisme causal est vraie, pour qu'il connaisse les mouvements passés et futurs de tous les corps, le démon de Laplace devrait non seulement connaître les positions de tous les corps, pour un instant donné, ainsi que toutes les forces dont la nature est animée, mais il devrait aussi savoir quelles sont les propriétés des corps qui génèrent des forces, quelles sont les lois qui régissent les forces, et quelles sont les lois générales du mouvement. $M$ ais même ces connaissances seraient insuffisantes, car le démon devrait également savoir qu'il n'existe aucun autre corps, aucune propriété additionnelle générant des forces, aucune loi additionnelle des forces, et aucune autre loi générale du mouvement. $C$ 'est seulement s'il était en possession de ces connaissances additionnelles que le démon serait en mesure de connaître les mouvements passés et futurs de tous les corps.

Terence H organ a bien saisi la nature du projet dans lequel le démon de Laplace devrait s'engager pour connaître les mouvements de tous les corps, en le caractérisant comme un calcul numérique cosmique². M ais si le démon de Laplace devait aussi connaître tous les faits du monde, alors il devrait s'engager dans un autre projet, soit celui del'herméneutique cosmique, pour reprendrel'expression de H organ ${ }^{3}$. Pour nous en tenir à l'essentiel, et en laissant de côté les subtilités de cette notion, I'herméneutique cosmique consiste à connaître tous les faits de notre monde à partir d'un sous-ensemble propre de ces faits - soit des faits de base si l'on peut dire. M a discussion dans ce texte porte principalement sur un projet d'herméneutique cosmique encore plus spécifique.

Laplace cherchait à caractériser le déterminisme causal (à tout le moins relativement au mouvement), mais dans ce qui suit, c'est un déterminismed'un autre ordre qui me préoccupe, à savoir le déterminisme physique, c'est-à-dire la thèse selon laquelle les faits physiques de notre monde déterminent tous les faits du monde. La doctrine du déterminisme physiqueque je veux discuter est, plus précisément, celle que Frank Jackson appelle « matérialisme», et qu'il formule de la façon suivante :

Toute réplique physique minimale de notre monde est une réplique simpliciter de notre monde 4 .

Une réplique simpliciter de notre monde est un monde possible qui est exactement comme notre monde sous tous ses aspects. Une réplique physique de notre monde est exactement comme notre monde sous tous ses aspects physiques : c'est un monde qui obéit exactement aux mêmes lois physiques que le nôtre, et qui a exactement le même nombre et les mêmes sortes d'objets et de champs que le nôtre, objets et champs qui sont exactement dans les mêmes états, qui sont impliqués dans les mêmes événements, et qui

2. Voir Horgan, 1983, p. 20, qui utilise l'expression « cosmic number crunching ».

3. Ibid., p. 20-21.

4. Jackson, 1998, p. 42. 
sont exactement dans les mêmes relations physiques que celles dans lesquelles se trouvent ceux de notre monde.

$M$ ais alors, qu'est-ce qu'une réplique physique minimale de notre monde? Pour être une réplique physique minimale de notre monde, un monde possible doit en être une réplique physiquequi ne contient pas plus de lois, d'objets, de champs, de propriétés, d'états, d'événements et de relations qu'il n'est requis pour qu'il soit une réplique physique de notre monde 5 . Bref, pour être une réplique physique minimale de notre monde, un monde doit en être une réplique physique qui ne contient strictement rien de plus que ce qui est requis pour en être une réplique physique.

Un monde peut être une réplique physique de notre monde sans pour autant en être une réplique physique minimale. Pour bien voir cela, il nous suffit d'abord de supposer que notre monde ne contient pas de fantômes, ce qui semble tout à fait inoffensif. Cependant, il pourrait bien y avoir une communauté de fantômes dans un monde qui constitue une réplique physique de notre monde, à condition que ces fantômes n'affectent, causalement, rien de physique dans ce monde. En effet, la totalité des faits physiques de notre monde n'entraîne pas, logiquement, l'inexistence d'une telle communauté de fantômes. Compte tenu de la totalité des faits physiques de notre monde, cette question demeure ouverte, d'un point de vue strictement logique. Puisqu'il n'existe pas une telle communauté de fantômes dans notre monde, comme nous venons de le supposer, et qu'il existe une telle communauté dans le monde possible en question, ce dernier est une réplique physique de notre monde, mais il n'en est pas une réplique physique minimale, puisqu'il contient plus qu'il n'est strictement requis pour être une réplique physique de notre monde, soit la communauté de fantômes en question. Pour prendre un exemple différent, supposons qu'un certain monde possible soit une réplique physique de notre monde, mais qu'en plus d'obéir aux lois physiques de notre monde, il obéisse à des lois additionnelles, qui ne sont pas des lois de notre monde. Cela serait possible à condition que ces lois additionnelles ne modifient en rien le cours des événements physiques de ce monde. Ainsi, alors que ce monde serait une réplique physique du nôtre, il n'en serait pas une réplique physique minimale.

Selon la doctrine du déterminisme physique, toute réplique physique minimale de notre monde est une réplique simpliciter de notre monde. En d'autres termes, toute réplique physique de notre monde qui ne contient rien de plus que ce qui est strictement requis pour être une réplique physique de

5. Je suppose que les catégories de lois, d'objets, de champs, de propriétés, d'états, d'événements et de relations (ou un certain sous-ensemble propre de ces catégories) sont les catégories fondamentales. S'il existe des catégories fondamentales additionnelles, nous n'avons qu'à les inclure dans cette liste. L'idée d'une réplique physique minimale est celle d'un monde qui ne contient que des occupants physiques pour chacune des catégories fondamentales d'ingrédients du monde. 
notre monde est exactement comme notre monde sous tous ses aspects, quels qu'ils soient. Cette doctrine a pour conséquence que notre monde est une réplique physique minimale de lui-même. Cela peut être clarifié de la façon suivante. Supposons que notre monde ne soit pas une réplique physique minimale de lui-même. De cela, il découle que notre monde contient quelque chose en plus de ce qui est strictement requis pour qu'il soit une réplique physique minimale de lui-même. De sorte qu'un monde pourrait être une réplique physique minimale de notre monde et ne pas en être une réplique simpliciter. Le déterminisme physique serait alors faux. N ous voyons donc que si notre monde n'est pas une réplique physique minimale de lui-même, alors le déterminisme physique est faux. Ce qui implique que si le déterminisme physique est vrai, alors notre monde est bien une réplique physique minimale de lui-même.

Évidemment, le déterminisme physique est une thèse empirique : si cette thèse est vraie, elle ne peut être connue qu'a posteriori. Q ue notre monde soit une réplique physique de lui-même est une vérité analytique, mais qu'il soit une réplique physique minimale delui-même, comme la doctrine du déterminisme physique l'implique, est une hypothèse empirique qui porte à controverse. Le fait que notre monde so it une réplique physique minimale de lui-même, si c'en est un, est un fait logiquement contingent qui n'est pas impliqué, logiquement, par la totalité des faits physiques, ni par la totalité des faits physiques en conjonction avec l'énoncé sel on lequel ces faits constituent la totalité des faits physiques de notre monde. Et c'est justement pour cela qu'une réplique physique de notre monde pourrait ne pas en être une réplique physique minimale.

II est clair que le déterminisme physique est une thèse discutable et sujette à controverse. S'il existe des faits mentaux, sociaux, éthiques et esthétiques, alors si la thèse du déterminisme physique est vraie, tout monde qui est une réplique physique minimale de notre monde devrait également contenir ces faits ${ }^{6}$. Car si cette thèse est vraie, alors toute réplique physique minimale du monde actuel doit contenir exactement les mêmes faits que celui-ci. En termes plus imagés, si le déterminisme physique est vrai, alors, pour créer un monde contenant tous les faits mentaux, sociaux, éthiques, et esthétiques de notre monde, la déesse-mère n'aurait qu'à créer un monde qui est une réplique physique minimale de notre monde. Son travail serait dès lors achevé : elle aurait ainsi créé un monde qui est une réplique simpliciter du nôtre et, par conséquent, un monde avec exactement les mêmes faits mentaux, sociaux, éthiques, et esthétiques que ceux de notre monde.

La thèse du déterminisme physique est-elle vraie? Est-il vrai, notamment, que toute réplique physique minimale du monde actuel doit être un monde contenant tous les faits mentaux, sociaux, éthiques, et esthétiques de notre monde? Je suis enclin à répondre positivement à ces questions, et j'ai

6. Voir Jackson, 1998, chap. 1. 
plutôt de la sympathie pour cette doctrine. Cela dit, mon but ici n'est pas de tenter de défendre cette doctrine de façon générale, mais c'est plutôt une des conséquences épistémiques du déterminisme physique qui me préoccupe, plus précisément la question suivante : si le déterminisme physique est vrai, alors qu'est-ce qu'un démon laplacéen devrait savoir s'il devait être en mesure de mener à bien le projet del'herméneutique cosmique?

Supposons que le déterminisme physique soit vrai, et supposons qu'un démon laplacéen connaîtrait tous les faits physiques de notre monde, c'està-dire tous les faits physiques nomologiques, et chacun des faits physiques particuliers et généraux de notre monde. La question est alors de savoir ce qu'il devrait connaître en plus de cela pour être en mesure de déduire tous les faits de notre monde.

D'abord, le démon devrait savoir que les faits physiques en question sont les seuls faits physiques de notre monde. $M$ ais il devrait également savoir que notre monde est une réplique physique minimale de lui-même. Sinon, le démon ne saurait pas, par exemple, s'il existe ou non une communauté de fantômes n'ayant aucun effet causal sur les faits physiques. $N$ ous voyons donc que s'il doit être en mesure de prédire tous les faits de notre monde, le démon devrait savoir que notre monde est une réplique physique minimale de lui-même.

De plus, puisque pour n'importe quelle affirmation possible visant à exprimer un fait le démon devrait savoir si ce qu'elle exprime est ou non un fait, le démon devrait posséder la connaissance requise pour comprendre n'importe laquelle de ces affirmations. Cela exigerait, notamment, que le démon possède la connaissance requise pour posséder tout concept nécessaire à la compréhension de chacune de ces affirmations possibles.

De plus, si le démon était lui-même situé dans le monde, il devrait également connaître certains faits indexicaux. Si, par exemple, j'étais moi-même le démon, je devrais savoir que je suis Brian M CLaughlin. Car c'est là une connaissance que je ne pourrais acquérir uniquement sur la base de ma connaissance de tous les faits physiques, même si on y ajoutait les connaissances additionnelles que je viens de noter, à savoir que ces faits physiques sont les seuls faits physiques, que notre monde est une réplique physique minimale de lui-même, ainsi que la connaissance de tout ce qui est requis pour comprendre toute affirmation possible sur le monde. En effet, sur la base de ces connaissances il me serait impossible de déduire que je suis Brian M CLaughlin. De la même manière, je devrais égal ement savoir que je suis maintenant à tel et tel endroit, à chaque moment où j'existe, tout commeje devrais savoir quel moment est le moment présent, pour chaque moment où j'existe.

II semble, à première vue, qu'en plus des connaissances que je viens d'énumérer, le démon aurait besoin d'en posséder certaines autres. Par exemple, c'est un fait quel'eau $=\mathrm{H}_{2} \mathrm{O}$. Or, quelqu'un pourrait ignorer ce fait même s'il comprenait l'affirmation que l'eau $=\mathrm{H}_{2} \mathrm{O}$. Car la vérité de cette affirmation ne peut être connue qu'a posteriori, de sorte que le démon devrait, 
semble-t-il, savoir quelles sortes de phénomènes macroscopiques sont identiques à quelles sortes de phénomènes microscopiques? ${ }^{7}$.

Il faut bien voir que l'idée selon laquelle le démon devrait connaître de telles identités théoriques est compatible avec le déterminisme physique. Cette idée serait incompatible avec le déterminisme physique seulement si de tels énoncés d'identité étaient vrais de façon contingente. Cependant, bien que les énoncés d'identité vrais ne puissent être connus qu'a posteriori, ils n'en sont pas moins nécessairement vrais car, comme Saul Kripke l'a montrée :

$\mathrm{Si} A=B$, alors il est nécessaire que $A=B$ (Principe de la nécessité del'identité).

$C^{\prime}$ 'est sur la base de l'indiscernabilité des identiques et de la thèse selon laquelle toute chose est nécessairement identique à elle-même que Kripke a proposé sa célèbre démonstration de ce principe. II a en outre expliqué clairement en quoi certains énoncés d'identité qui, à première vue, sembleraient être des contre-exemples à ce principe - comme par exemple «Benjamin Franklin est l'inventeur des lunettes à double foyer » - n'en sont pas, dans la mesure où les descriptions sont souvent utilisées de façon non rigide pour désigner différents individus dans différentes circonstances possibles. Comme il l'a démontré, lorsque les termes singuliers de chaque côté du signe d'identité sont des désignateurs rigides, alors si l'énoncé d'identité est vrai, il sera nécessairement vrai. O r, les termes de sortes macroscopiques, comme le terme « eau », par exemple, sont des désignateurs rigides, tout commele sont les descriptions comme « $\mathrm{H}_{2} \mathrm{O}$ ». Q ue l'eau soit identique à I' $\mathrm{H}_{2} \mathrm{O}$ est vrai dans tous les mondes possibles où l'eau existe. Et, de façon plus générale, la connaissance d'identités théoriques n'est pas une connaissance de faits contingents, bien qu'elle soit a posteriori. Pour cette raison, la thèse selon laquelle le démon devrait connaître les identités théoriques est compatible avec la doctrine du déterminisme physique.

Considérons maintenant les faits relatifs aux caractères phénoménologiques des expériences, c'est-à-dire les faits concernant les aspects de ce que cela fait que d'avoir certaines expériences, comme par exemple les faits relatifs à ce que cela fait que defairel'expérience d'une douleur intense. S'il y a de tels faits, alors si le déterminisme physique est vrai le démon laplacéen devrait être capable de les prédire. A ppelons cette sorte de faits des faits d'expérience. Si l'une ou l'autre des thèses du behaviorisme analytique ou du fonctionnalisme analytiqueétait vraie des expériences, le démon serait en mesure de prédire les faits d'expérience à partir des connaissances suivantes : i) sa connaissance de la totalité des faits physiques, ii) sa connaissance que cette totalité est la totalité des faits physiques, iii) sa connaissance que notre monde est une réplique physique minimale de lui-même, iv) sa connaissance des faits indexicaux à propos de lui-même (s'il se trouve localisé dans le monde), et finalement v) sa compré-

7. Voir H organ, 1983, p. 23-24.

8. Voir Kripke, 1980. 
hension de toute affirmation visant à exprimer des faits d'expérience. $M$ ais, malheureusement pour le déterministe physique, ces deux thèses sont fausses quant aux faits d'expérience ${ }^{9}$. Par conséquent, les connaissances que nous venons de noter seraient insuffisantes pour quele démon soit en mesure de prédire les faits d'expérience de notre monde.

$\mathrm{Si}$, pour prédire les faits d'expérience de notre monde, le démon devait en outre connaître certaines lois psychophysiques selon lesquelles certains états physiques produisent certains états d'expérience, alors le déterminisme physique serait faux. M ais si, par contre, les caractères phénoménologiques des expériences sont identiques à certaines propriétés physiques ou à certaines propriétés fonctionnelles, alors en connaissant ces identités le démon pourrait prédire les faits d'expérience. La connaissance de ces identités serait a posteriori, mais elle n'en serait pas moins une connaissance de vérités nécessaires et, ainsi, le fait qu'une telle connaissance soit requise pour mener à bien le projet del'herméneutique cosmique serait compatible avec le déterminisme physique.

Convenons d'utiliser l'expression « connaissance des faits physiques de notre monde » en un sens large inhabituel comme une abréviation qui inclut la connaissance des faits physiques de notre monde, la connaissance que ces faits sont les seuls faits physiques de notre monde, et la connaissance que notre monde est une réplique physique minimale de lui-même. II faut bien noter cependant qu'une telle expression peut être trompeuse, dans la mesure où si le fait que notre monde soit une réplique physique minimale de lui-même peut être appelé un fait physique, il s'agit tout de même d'un fait physique d'une sorte bien particulière. Généralement, les faits physiques sont ceux qui découlent logiquement de la théorie physique qui est vraie de notre monde. Cependant, une telle théorie physique n'entraîne pas que notre monde soit une réplique physique minimale de lui-même, puisque la théorie physique qui est vraie de notre monde est également vraie de toute réplique physique de notre monde; et, comme nous I'avons noté, un monde peut être une réplique physique de notre monde sans en être une réplique physique minimale. $M$ ais si nous gardons à l'esprit cette distinction importante, il ne devrait plus y avoir de danger de tromper qui que ce soit en utilisant l'expression « connaissance des faits physiques de notre monde » dans le sens élargi que nous venons de stipuler, à savoir comme une abréviation qui inclut la connaissance que notre monde est une réplique physique minimale de lui-même.

D avid Chalmers et Frank Jackson ont récemment soutenu un certain point de vue qui implique que la connaissance d'identités théoriques portant sur des phénomènes non mentaux - comme par exemple quel'eau $=\mathrm{H}_{2} \mathrm{O}-$

9. Ces deux thèses sont peut-être fausses également de tout type de faits mentaux, mais c'est là une question que je laisse ouverte. Pour des arguments contre le fonctionnalisme analytique, qui me paraissent convaincants (et qui s'appliquent également au behaviorisme analytique), voir notamment $\mathrm{H}$ ill, 1991, et Chalmers, 1996. 
est une connaissance que le démon pourrait obtenir sur la seule base de la connaissance physique que nous venons de noter, à condition que celle-ci soit complétée par la connaissance requise pour comprendre toute affirmation possible visant à exprimer de telles identités théoriques ${ }^{10}$. J ackson et $\mathrm{Chal-}$ mers reconnaissent qu'on peut comprendre l'affirmation que l'eau $=\mathrm{H}_{2} \mathrm{O}$ sans savoir si elle est vraie. $M$ ais, ils maintiennent qu'il serait possible de savoir qu'elle est vraie sur la base de la connaissance requise pour comprendre pleinement toute affirmation possible visant à exprimer des faits, et de la connaissance des faits physiques, dans le sens large que nous venons de stipuler au paragraphe précédent.

Leur raison de soutenir que le démon pourrait connaître des faits comme le fait que l'eau $=\mathrm{H}_{2} \mathrm{O}$ sur cette seule base épistémique est la suivante. M êmesi le concept d'eau n'admet pas une définition a priori en termes microphysiques ou en termes neutres (topic neutral), il n'en est pas moins associé a priori à une description qui en fixe la référence de façon contingente. U ne telle description qui fixe la référence correspond approximativement, selon eux, à quelque chose qui ressembleà la description suivante : « le liquide transparent, potable, qui tombe du ciel quand il pleut, qui remplit les océans, les fleuves, les ruisseaux, etc. », même si la façon exacte dont la description devrait être complétée demeure incertaine. Et Chalmers utilise l'expression « la substance aqueuse ${ }^{11}$ » comme une abréviation d'une telle description qui fixe la référence. II est important de noter que la description « la substance aqueuse » est une description non rigide (tout comme la description «l'inventeur des lunettes à double foyer »), c'est-à-dire que ce qui satisfait cette description, ce qui compte comme substance aqueuse, peut varier d'un monde possible à un autre. Cependant, on peut facilement rigidifier cette description en y ajoutant tout simplement le mot « actuelle ». Ainsi, la description « la substance aqueuse actuelle » est bien une description rigide, et c'est une vérité nécessaire que l'eau = la substance aqueuse actuelle. L'affirmation que l'eau = la substance aqueuse actuelle correspond à une sorte d'analyse fonctionnelle du concept d'eau. Or, selon Jackson et Chalmers, la connaissance de l'analyse fonctionnelle du concept d'eau serait requise pour avoir une possession complète du concept d'eau, et par conséquent elle serait requise pour une compréhension complète de l'affirmation que l'eau $=\mathrm{H}_{2} \mathrm{O}$. Ils soutiennent que si le démon connaissait tous les faits physiques de notre monde et s'il savait que notre monde est une réplique physique minimale de lui-même, il serait alors en mesure de savoir que l'eau $=\mathrm{H}_{2} \mathrm{O}$, puisqu'il saurait que l'eau $=$ la substance aqueuse actuelle, dans la mesure où une telle connaissance est a priori ${ }^{12}$. De plus, le démon

10. Voir Chalmers, 1996, et Jackson, 1998.

11. Nous traduisons ainsi l'expression anglaise "the watery stuff», utilisée par Chalmers (note de la traduction).

12. Voir Jackson, 1998, chap. 2, et Chalmers, 1996, p. 78-79. 
serait en mesure de faire l'inventaire de tous les candidats actuels qui correspondent à la substance aqueuse, et il pourrait savoir qu'il s'agit là de la totalité des candidats actuels. Sur la base de cette connaissance, il pourrait tout simplement déduire que $\mathrm{I}^{\prime} \mathrm{H}_{2} \mathrm{O}$ est la seule substance aqueuse actuelle. Puisqu'il saurait a priori que l'eau = la substance aqueuse actuelle, le démon serait capable de prédire que l'eau $=\mathrm{H}_{2} \mathrm{O}$. Par conséquent, il pourrait mener à bien le projet de l'herméneutique cosmique sans devoir connaître à l'avance les identités théoriques comme l'eau $=\mathrm{H}_{2} \mathrm{O}$. Si J ackson et Chalmers ont raison en ce qui concerne les concepts macroscopiques de sortes naturelles, comme le concept d'eau, alors, si le déterminisme physique est vrai, le démon sera en mesure de déduire les identités macrophysiques/microphysiques à partir de sa connaissance des faits physiques de notre monde et de sa compréhension des affirmations exprimant de telles identités.

Cette idée de Jackson et Chalmers, sel on laquelle les concepts de sortes naturelles, comme le concept d'eau, seraient associés a priori à des conditions qui en fixent la référence de façon contingente, me paraît relativement plausible. Si de tels concepts ont effectivement des conditions a priori contingentes qui en fixent la référence, alors il est tout à fait plausible que la connaissance de ces conditions soit requise pour avoir une compréhension complète de ces concepts. II me suffit de noter que J ackson et Chalmers appuient de façon assez convaincante l'affirmation voulant que le démon serait en mesure de savoir que l'eau $=\mathrm{H}_{2} \mathrm{O}$ uniquement à partir de sa connaissance des faits physiques (au sens large noté plus haut), et de la connaissance requise pour avoir une compréhension complète des affirmations exprimant de telles identités. Q uoi qu'il en soit, c'est là une thèse que je n'ai pas l'intention de contester.

Comme Chalmers le souligne, contrairement aux concepts macroscopiques de sortes naturelles, comme le concept d'eau, les concepts phénoménaux - c'est-à-dire les concepts exprimant le caractère phénoménal des expériences - ne sont pas associés a priori à des descriptions qui en fixeraient la référence de façon contingente ${ }^{13}$. La condition qui fixe, par exemple, la référence du concept phénoménal de douleur - c'est-à-dire le concept de la qualité phénoménale de douleur - est la qualité phénoménale elle-même telle qu'elle est ressentie, et non pas un mode de présentation contingent de la propriété. Ainsi, les concepts phénoménaux n'admettent pas d'analyses fonctionnelles. Le démon pourrait très bien comprendre, par exemple, que la douleur = l'activation des fibres- $C$ sans être en mesure de déterminer, sur la base de sa connaissance des faits physiques de notre monde, si cette affirmation d'identité est vraie (même si cette connaissance est entendue dans le sens élargi que nous avons noté plus haut). De façon plus générale, selon le point de vue de Chalmers, le démon ne pourrait connaître aucun fait d'expérience uniquement sur la base de sa connaissance des faits physiques de notre monde et

13. Voir Chalmers 1996, chap. 2. 
de sa compréhension des affirmations visant à exprimer des faits d'expérience. Cette compréhension et cette connaissance des faits physiques ne lui fourniraient pas une base à partir de laquelle il pourrait déduire un fait d'expérience, quel qu'il soit.

Je suis d'accord avec cette idée de Chalmers selon laquelle les concepts phénoménaux, comme le concept de la qualitéressentie de la douleur, ne sont pas associés a priori et de façon contingente à des conditions qui en fixent la référence ; je suis aussi d'accord avec l'idée que la condition qui fixe la référence du concept phénoménal de douleur est la qualitéressentie dela douleur elle-même ${ }^{14}$. Et, j'accepte égal ement l'idée que le démon ne pourrait connaître aucun fait d'expérience de notre monde uniquement sur la base de sa connaissance de tous les faits physiques du monde (dans notre sens élargi), et de la compréhension des affirmations exprimant des faits d'expérience.

Cela dit, je suis en désaccord avec le point de vue de C halmers concernant ce que le démon devrait savoir pour connaître les faits d'expérience de notre monde. La position de Chalmers relativement au projet d'herméneutique cosmique qui nous intéresse ici est la suivante: selon lui, pour mener à bien le projet d'herméneutique cosmique, le démon devrait connaître tous les faits physiques de notre monde, il devrait posséder la connaissance requise pour comprendre toute affirmation possible sur notre monde, il devrait aussi connaître certains faits indexicaux, s'il était localisé dans notre monde, et, finalement, il devrait savoir quels sont les divers faits physiques ou fonctionnels qui sont nomologiquement suffisants pour les divers faits d'expérience. A insi, selon ce point de vue, le démon devrait connaître des lois liant les faits physico-fonctionnels aux faits d'expérience, c'est-à-dire des lois phénoméno/ physico-fonctionnelles. Si Chalmers a raison de soutenir que le démon devrait connaître de telles lois, alors le déterminisme est faux. Car ces lois ne seraient pas déterminées par les lois physiques et les faits physiques de notre monde. Et, par conséquent, il serait faux de dire que toute réplique physique minimale de notre monde en est une réplique simpliciter, puisque les lois phénoméno/physico-fonctionnelles de notre monde seraient différentes de celles de toute réplique physique minimale de notre monde, pour la simple raison que de telles lois feraient défaut à toute réplique physique minimale de notre monde. Chalmers soutient qu'une réplique physique minimale de notre monde serait dépourvue de faits de conscience; les contreparties de nousmêmes dans un tel monde seraient des « zombies ${ }^{15}$ ». II soutient donc que le déterminisme physique est faux.

Je pense que Chalmers a tort. Le démon n'a pas besoin de connaître des lois phénoméno/physico-fonctionnelles pour connaître les faits d'expérience

14. Dans la terminologie technique de Chalmers, 1996, chap. 2, I'intension primaire et I'intension secondaire du concept de la qualité ressentie de la douleur coïncident. Je suis d'accord avec ce point.

15. Ibid., chap. 3. 
de notre monde. Selon moi, tout ce dont il aurait besoin serait de connaître toutes les affirmations d'identités phénoméno/physico-fonctionnelles vraies, et il devrait savoir que celles-ci constituent les seules affirmations d'identités phénoméno/physico-fonctionnelles vraies. $M$ ais, comme je l'ai noté, le fait que ce type de connaissance soit requis pour mener à bien le projet d'herméneutique cosmique est compatible avec le déterminisme physique, car de telles affirmations d'identité utilisent des désignateurs rigides et sont, par conséquent, nécessairement vraies, même si elles sont a posteriori. Dans la mesure où elles sont nécessairement vraies, le fait que le démon doit connaître de telles identités est compatible avec le déterminisme physique. De plus, il est très facile de comprendre pourquoi le démon devrait connaître de telles identités. Premièrement, elles ne peuvent être connues qu'a posteriori. D euxièmement, elles ne peuvent être déduites a priori à partir de la totalité des faits physiques (dans notre sens élargi), et de la connaissance requise pour comprendre ces faits. Troisièmement, la raison pour laquelle ces identités ne peuvent être ainsi déduites a priori est que, contrairement aux macroconcepts ordinaires comme celui d'eau, les concepts phénoménaux n'admettent pas d'analyses fonctionnelles en termes physiques ou en termes neutres (topic neutral).

Chalmers a anticipé une objection similaire, et il maintient que quelqu'un qui, pour défendre le déterminisme physique, endosse une position comme celle que je viens de présenter serait engagé à soutenir une thèse qu'il appelle « la nécessité métaphysique forte ». II caractérise cette dernière de la façon suivante:

Selon cette thèse, il y a moins de mondes métaphysiquement possibles qu'il n'y a de mondes logiquement possibles, et la nécessité a posteriori de certaines affirmations peut découler de facteurs qui sont indépendants de la sémantique des termes impliqués ${ }^{16}$.

J'ignore si quelqu'un soutient effectivement cette thèse de la nécessité métaphysique forte. $M$ ais, quoi qu'il en soit, ce n'est pas une thèse que j'endosse. Je pense que les mondes métaphysiquement possibles sont tout simplement identiques aux mondes logiquement possibles. Et la nécessité a posteriori des identités phénoméno/physico-fonctionnelles doit être expliquée sur la base de la sémantique des termes qui se trouvent de chaque côté du signe d'identité. Le fait que ces termes soient des désignateurs rigides devra apparaître dans une explication de la raison pour laquelle ces identités sont nécessairement vraies, si elles sont vraies. De plus, le fait qu'un des deux termes exprime un concept phénoménal, alors quel'autre exprime un concept physico-fonctionnel, devra apparaître dans une explication de la raison pour laquelle de telles identités ne peuvent être connues qu'a posteriori, bien qu'elles soient nécessaires. Car les concepts phénoménaux ne sont pas définissables en termes de

16. Ibid., p. 137. 


\section{Philosophiques / Printemps 2000}

concepts physico-fonctionnels; et, de plus, les concepts phénoménaux n'admettent pas même d'analyses fonctionnelles en termes physico-fonctionnels. C'est d'ailleurs pour cette raison qu'il existe un fossé explicatif, selon l'expression de Joseph Levine, entre le phénoménal et le physico-fonctionnel : les faits et les propriétés d'expérience ne peuvent avoir d'explications réductrices, que ce soit via des définitions ou via des analyses fonctionnelles ${ }^{17}$. Les concepts phénoménaux sont spéciaux. C'est d'ailleurs pour cette raison que le problème corps-esprit est un puzzle aussi déroutant depuis D escartes. C ependant, le déterministe physique est en mesure de soutenir que mêmesi les concepts phénoménaux sont spéciaux, les états et les propriétés d'expérience ne le sont pas. Le dualisme conceptuel n'implique pas le dualisme ontologique. Le déterministe physique peut soutenir que de telles affirmations d'identité sont vraies, et nécessairement vraies. Puisque si elles sont vraies, elles le sont nécessairement, le fait que le démon doive connaître de telles identités, pour mener à bien le projet d'herméneutique cosmique, ne pose aucune difficulté à la doctrine du déterminisme physique.

Ce qui sépare un déterministe physique de Chalmers est la question de savoir si les identités phénoméno/physico-fonctionnelles peuvent être vraies. Chalmers semble soutenir qu'elles ne le peuvent pas, et cela sur la base des trois raisons suivantes : (1) si elles sont vraies, alors elles sont nécessairement vraies; (2) nous pouvons concevoir de façon cohérente qu'elles soient fausses ; et (3) la concevabilité cohérente impliquela possibilité, sauf lorsque I'apparence de contingence d'une affirmation d'identité peut être expliquée en montrant que la personne qui prétend concevoir la fausseté d'une telle affirmation aurait confondu le phénomène en question avec des conditions qui fixent, de façon contingente, la référence de l'un ou l'autre des termes. J'accepte (1) et (2), mais je rejette (3). Chalmers ne nous a donné aucune raison convaincante de croire que les identités phénoméno/physico-fonctionnelles ne peuvent être vraies.

Le lien entre la concevabilité et la possibilité est plus faible que celui de conséquence logique. Le fait que $P$ soit concevable est une raison prima facie de croire qu'il est possible que $\mathrm{P}^{18}$. $\mathrm{M}$ ais une telle raison prima facie peut être révoquée. Le déterministe physique peut maintenir que la raison prima faciede croire, par exemple, que la corrélation entre la douleur et un certain état physico-fonctionnel corrélatif n'est que contingente peut être révoquée sur la base des principes de simplicité théorique et de cohérence générale. II peut justifier les identités phénoméno/physico-fonctionnelles, dans la mesure où elles offrent la meilleure explication de ces corrélations. De plus, même si j'accepte que nous ne puissions expliquer l'apparence de contingence des affirmations d'identités phénoméno/physico-fonctionnelles de la façon décrite en (3), cela n'est pas la seule façon d'expliquer cette apparence de contingence. Par exem-

17. Voir Levine, 1983, 1998.

18. Voir Yablo, 1993. 
ple, dans le cas de l'affirmation que la douleur = l'activation des fibres- $C$, une telle apparence de contingence s'expliquera du fait que le concept de douleur a un rôle conceptuel très différent de celui du concept d'activation des fibres$C$, un rôle conceptuel si différent qu'il ne peut y avoir aucun lien a priori entre ces concepts ${ }^{19}$.

En conclusion, si le déterminisme physique est vrai, alors, pour mener à bien le projet d'herméneutique cosmique, le démon de L aplace devrait connaître les choses suivantes : la totalité des faits physiques de notre monde, le fait que ces faits physiques constituent la totalité des faits physiques de notre monde, le fait que notre monde est une réplique physique minimale de luimême, tout ce qui est requis pour comprendre toute affirmation possible sur notre monde, des faits indexicaux sur le démon lui-même, et, finalement, des identités phénoméno/physico-fonctionnelles. Bien sûr, la question de savoir si ces connaissances seraient une base suffisante pour connaître les faits sociaux, éthiques, et esthétiques de notre monde reste ouverte. J'ai dela sympathie pour l'idée qu'elles le seraient, mais la défense de cette thèse devra attendre une autre occasion ${ }^{20}$.

\section{Bibliographie}

Chalmers, D., 1996. The Conscious M ind, O xford, O xford University Press. Hill, C., 1991. Sensations: A D efense of Type Materialism, Cambridge, Cambridge University Press.

Hill, C., 1997. "Imaginability, Conceivability, Possibility and the M indBody Problem », Philosophical Studies, 87, p. 61-85.

Hill, C. et M CLaughlin, B. P., 1999. "There Are Fewer Things Than Are Dreamt of in Chalmer's Philosophy », Philosophy and Phenomenological Research, 59, p. 445-454.

H organ, T., 1983. "Supervenience and Cosmic Hermeneutics», Southern Journal of Philosophy, suppl. 22, p. 19-38.

Jackson, F., 1998. From M etaphysics to Ethics: A D efense of Conceptual A nalysis, $\mathrm{N}$ ew York, $\mathrm{O}$ xford University Press.

Kripke, S., 1980. Naming and Necessity, Cambridge (M ass.), $\mathrm{H}$ arvard University Press.

Laplace, P.-S., 1886. Théorie analytique des probabilités, Tome VII des $O$ euvres complètes de $L$ aplace, $3^{\mathrm{e}}$ édition publiée sous les auspices de I'A cadémie des Sciences, par M M. les secrétaires perpétuels, Paris, Gauthier-Villars, 1878-1912.

Levine, J., 1983. « M aterialism and Q ualia: The Explanatory Gap », Pacific Philosophical Q uarterly, 64, p. 354-361.

19. Voir Hill, 1997, Hill et MCLaughlin, 1999, et M CLaughlin, à paraître, pour un développement et une défense des points proposés dans ce paragraphe.

20. Ce texte a été traduit de l'anglais par Paul Bernier. 
Levine, J., 1998. "C onceivability and The M etaphysics of M ind », N ous, 32, p. 449-480.

M CLaughlin, B. P., (à paraître). "In D efense of $\mathrm{N}$ ew Wave $\mathrm{M}$ aterialism », dans Loewer, Barry, et Gillett, Carl, dir., Physicalism and Its $D$ iscontents, Cambridge, Cambridge University Press.

Yablo, S., 1993. « Is Conceivability a Guide to Possibility? », Philosophy and Phenomenological Research, 53, p. 1-42. 